\section{Anti-apoptotic BCL2L2 increases megakaryocyte proplatelet formation in cultures of human cord blood}

\author{
Seema Bhatlekar, ${ }^{1}$ Indranil Basak, ${ }^{1}$ Leonard C. Edelstein, ${ }^{2}$ Robert A. \\ Campbell, ${ }^{1}$ Cory R. Lindsey, ${ }^{2}$ Joseph E. Italiano Jr., ${ }^{3}$ Andrew S. Weyrich, ${ }^{1}$ Jesse \\ W. Rowley, ${ }^{1}$ Matthew T. Rondina, ${ }^{1,4}$ Martha Sola-Visner ${ }^{5}$ and Paul F. Bray ${ }^{1,6}$
}

${ }^{1}$ Program in Molecular Medicine and Department of Internal Medicine, University of Utah, Salt Lake City, UT; ${ }^{2}$ Cardeza Foundation for Hematologic Research, Thomas Jefferson University, Philadelphia, PA; ${ }^{3}$ Brigham and Women's Hospital, Harvard University, Boston, MA; ${ }^{4}$ George E. Wahlen VAMC GRECC, Salt Lake City, UT; ${ }^{5}$ Boston Children's Hospital, Harvard University, Boston, MA and ${ }^{6}$ Division of Hematology and Hematologic Malignancies, Department of Internal Medicine, University of Utah, Salt Lake City, UT, USA

\section{ABSTRACT}

A poptosis is a recognized limitation to generating large numbers of megakaryocytes in culture. The genes responsible have been rigorously studied in vivo in mice, but are poorly characterized in human culture systems. As CD34-positive $\left(^{+}\right)$cells isolated from human umbilical vein cord blood were differentiated into megakaryocytes in culture, two distinct cell populations were identified by flow cytometric forward and side scatter: larger size, lower granularity (LLG), and smaller size, higher granularity (SHG). The LLG cells were CD41a $\mathrm{a}^{\text {High }}$ CD42 $\mathrm{a}^{\text {High }}$ phosphatidylserine ${ }^{\text {Low }}$, had an electron microscopic morphology similar to mature bone marrow megakaryocytes, developed proplatelets, and displayed a signaling response to platelet agonists. The SHG cells were $\mathrm{CD} 41 \mathrm{a}^{\text {Low }} \mathrm{CD} 42 \mathrm{a}^{\text {Low }}$ phosphatidylserine ${ }^{\text {High }}$, had a distinctly apoptotic morphology, were unable to develop proplatelets, and showed no signaling response. Screens of differentiating megakaryocytes for expression of 24 apoptosis genes identified $B C L 2 L 2$ as a novel candidate megakaryocyte apoptosis regulator. Lentiviral $B C L 2 L 2$ overexpression decreased megakaryocyte apoptosis, increased CD $41 \mathrm{a}^{+}$LLG cells, and increased proplatelet formation by $58 \%$. An association study in 154 healthy donors identified a significant positive correlation between platelet number and platelet BCL2L2 mRNA levels. This finding was consistent with the observed increase in platelet-like particles derived from cultured megakaryocytes over-expressing BCL2L2. BCL2L2 also induced small, but significant increases in thrombin-induced platelet-like particle $\alpha \operatorname{IIb} \beta 3$ activation and Pselectin expression. Thus, BCL2L2 restrains apoptosis in cultured megakaryocytes, promotes proplatelet formation, and is associated with platelet number. BCL2L2 is a novel target for improving megakaryocyte and platelet yields in in vitro culture systems.

\section{Introduction}

Hematopoietic stem cells (HSC) are essential for reconstituting hematopoiesis in the setting of bone marrow (BM) transplantation and are of great value for studies of the basic biology of hematopoiesis. Differentiating HSC into megakaryocytes $(\mathrm{MK})$ in culture has become a standard approach to study megakaryocytopoiesis (MKpoiesis). MK culture systems are also powerful tools for the functional assessment of novel platelet genes, gene variants, protein-coding transcripts, and microRNA associated with platelet reactivity and clinical hemorrhagic or thrombotic disorders. Recent advancements in MKpoiesis research have opened up a new, promising field of ex vivo manufacturing of platelets, ${ }^{1-4}$ with the ultimate goal of infusing these products into patients with thrombocytopenia or qualitative
Ferrata Storti Foundation
Haematologica 2019
Volume 104(10):2075-2083

\section{Correspondence:}

PAUL F. BRAY

paul.bray@hsc.utah.edu

Received: August 16, 2018.

Accepted: January 30, 2019.

Pre-published: February 7, 2019.

doi:10.3324/haematol.2018.204685

Check the online version for the most updated information on this article, online supplements, and information on authorship \& disclosures: www.haematologica.org/content/104/10/2075

(C)2019 Ferrata Storti Foundation

Material published in Haematologica is covered by copyright. All rights are reserved to the Ferrata Storti Foundation. Use of published material is allowed under the following terms and conditions:

https://creativecommons.org/licenses/by-nc/4.0/legalcode. Copies of published material are allowed for personal or internal use. Sharing published material for non-commercial purposes is subject to the following conditions:

https://creativecommons.org/licenses/by-nc/4.0/legalcode, sect. 3. Reproducing and sharing published material for commercial purposes is not allowed without permission in writing from the publisher. 
platelet disorders. Improvements in this technology may also allow 'designer' platelets to be engineered that overcome immunological incompatibility and infection issues. ${ }^{5}$

A major limitation of all MK culture systems is the relatively short time period for which the differentiating $M K$ can be kept viable before they die through apoptosis. ${ }^{6}$ The role of apoptosis during MKpoiesis is somewhat controversial, , with some data supporting a role for the intrinsic pathway of apoptosis in platelet production, ${ }^{8-10}$ while other studies show that MK must restrain apoptosis to survive and progress safely through proplatelet formation and platelet generation. ${ }^{11-13}$ However, the apoptosis regulators of human cord blood-derived (CB)-MK cultures remain poorly understood. During the course of our studies with cultured CB MK, we observed two distinct populations of cells by forward and side scatter flow cytometry. The aims of the current study were to determine: (i) whether there were viability and apoptosis differences between these two populations; and (ii) molecular mechanism(s) of apoptosis regulation during Mkpoiesis. We also wanted to begin to identify approaches that would reduce $\mathrm{MK}$ apoptosis in order to produce greater yields of $\mathrm{MK}$ and platelet in cultures. We demonstrate that the anti-apoptosis Bcl2 family member BCL2L2 (encoding Bcl$w)$ regulates cultured $M K$ apoptosis, promotes proplatelet formation, and is associated with human platelet number.

\section{Methods}

\section{Primary megakaryocyte cultures}

Human umbilical cord CB was obtained from the New York Blood Center (New York, NY, USA) under institutional review board (IRB) approval (00108527). CD34 $4^{+}$hematopoietic stem and progenitor cells (HSPC) were isolated from human umbilical vein $\mathrm{CB}$ and cultured in serum free expansion media supplemented with $25 \mathrm{ng} / \mathrm{mL}$ of stem cell factor (SCF) and $20 \mathrm{ng} / \mathrm{mL}$ of thrombopoietin (TPO) (Peprotech, Rocky Hill, NJ, USA) for six days. Cells were cultured with $50 \mathrm{ng} / \mathrm{mL}$ TPO only from days $6-13 .{ }^{14}$ Adult granulocyte-macrophage colony-stimulating factor (GMCSF) mobilized CD34 HSPC were purchased from the Utah Cell Therapy and Regenerative Medicine Center (Salt Lake City, UT, USA) under the University of Utah IRB approval (00108527).

\section{Megakaryocyte proplatelet formation assay}

Day 9 transduced cells were plated at $2 \times 10^{4}$ cells $/ \mathrm{mL}$ in $60 \mu$-Dish Grid-500 plates (Ibidi, Fitchburg, WI, USA) using fresh medium supplemented with $50 \mathrm{ng} / \mathrm{mL}$ TPO. On day 13, the proplatelet forming (PPF) MK, defined as displaying at least one filamentous pseudopod, were scored with a light microscope blinded as to experimental group. Images were taken at room temperature under 40x objective, numerical aperture 1.35, using FV1000 confocal laser scanning microscope (Olympus, Center Valley, PA, USA). The percentage of PPF MK was calculated as the number of PPF MK compared to the total number of round cultured cells analyzed. An average 200 cells were counted per condition.

\section{Integrin $\alpha$ llb $\beta 3$ activation assessment}

Cells were resuspended in Tyrode's buffer ( $138 \mathrm{mM} \mathrm{NaCl}, 5.5$ $\mathrm{mM}$ dextrose, $12 \mathrm{mM} \mathrm{NaHCO}_{3}, 0.8 \mathrm{mM} \mathrm{CaCl}_{2}, 0.4 \mathrm{mM} \mathrm{MgCl}_{2}$, $2.9 \mathrm{mM} \mathrm{KCl}_{2}, 0.36 \mathrm{mM} \mathrm{Na}_{2} \mathrm{HPO}_{4}, 20 \mathrm{mM}$ Hepes, pH 7.4). Integrin $\alpha I l b \beta 3$ activation was quantified with FITC-labeled PAC1 (1:100) (BD Pharmingen) in response to stimulation ${ }^{15}$ with no agonist (resting), $100 \mu \mathrm{M}$ PAR4-AP [GL Biochem (Shanghai) Ltd., China], $250 \mathrm{nM}$ thrombin (Enzyme Research, South Bend,
IN, USA) or $10 \mu \mathrm{g} / \mathrm{mL}$ CRP (synthesized at Baylor College of Medicine and cross-linked with glutaraldehyde) for 20 minutes (min) at $37^{\circ} \mathrm{C}$, followed by $4 \%$ paraformaldehyde fixation at room temperature. Cells were analyzed on a Cytoflex or BD Accuri C6 flow cytometer.

\section{Statistical analysis}

All statistical analyses were performed using GraphPad Prism 6 software version 10.1 (La Jolla, CA, USA) and reported as Mean \pm Standard Error of Mean (SEM). Fold changes for BCL2L2 levels over time in cultures and for lentiviral overexpression were presented as $\log _{2}$ (fold change) compared to their respective controls (day 6 for changes in BCL2L2 levels over time and empty vector control for overexpression) and analyzed by a one-sample ttest. $\log _{2}$ transformation was adopted to have a normally distributed fold-change data, determined by Kolmogorov-Smirnov normality test. The relationship between platelet BCL2L2 mRNA expression levels and platelet counts in the 154 healthy human donors in the Platelet RNA Expression Study 1 (PRAX-1) was assessed by Pearson's correlation with 95\% Confidence Interval $(95 \% \mathrm{CI})$. For all other analyses, statistical significance was assessed using paired Student $t$-test. $P<0.05$ was considered statistically significant.

See Online Supplementary Appendix for further details of the experiments.

\section{Results}

\section{Subsets of cultured megakaryocytes show differing viability}

CD34 ${ }^{+}$cells were isolated from human umbilical vein $\mathrm{CB}$ and cultured to generate $\mathrm{MK}$ as described previously. ${ }^{14}$ By day $13, \mathrm{CD}^{+} 4^{+}$cells were differentiated into $\mathrm{MK}$, as demonstrated by the expression of mature MK markers (Online Supplementary Figure S1A-D). Day 13 MK were larger than day 0 HSC (Online Supplementary Figure S1E) and demonstrated polyploid MK (Online Supplementary Figure S1F and G). Thus, these culture conditions promoted generation of mature, polyploid MK.

Flow cytometric analysis of day 13 cultures revealed distinct cell populations differing by forward scatter (FSC) and side scatter (SSC) characteristics (Figure 1A): larger MK with lower granularity [larger, lower granular (LLG)] and smaller MK with higher granularity [smaller, higher granular (SHG)]. Peripheral blood (PB) mobilized adult CD34 $4^{+}$cells cultured under the same conditions also generated similar subpopulations of MK (Online Supplementary Figure S2). Approximately 80\% of LLG MK expressed both $\mathrm{CD} 41 \mathrm{a} / \mathrm{CD} 42 \mathrm{a}$ and $\mathrm{CD} 41 \mathrm{a} / \mathrm{CD} 42 \mathrm{~b}$ markers, while only $20-30 \%$ SHG MK were double positive for mature MK markers (Figure 1B). Lower surface expression of CD42b (GPIba) in day 13 SHG cells compared to LLG cells is consistent with mitochondrial damage ${ }^{16}$ as observed in apoptotic adult mobilized HSPC-derived MK. ${ }^{17}$

Since the numbers of SHG MK showed less differentiation towards mature MK than the LLG MK, and because SHG MK showed reduced CD42b expression, we considered whether SHG MK may have undergone (or be undergoing) apoptosis. Figure $1 \mathrm{C}$ shows few $\mathrm{CD} 41 \mathrm{a}^{+} \mathrm{LLG}$ MKbound annexin $\mathrm{V}$ [termed phosphatidylserine (PS) ${ }^{\mathrm{Low}}$ ], a marker of apoptosis-induced phosphatidylserine expression, whereas a significantly higher percentage of CD $41 \mathrm{a}^{+}$ SHG MK bound annexin V (termed PS ${ }^{\text {High }}$ ). Anti-annexin 
V microbeads were used to separate day 13 PS ${ }^{\text {Low }}$ LLG MK from PS ${ }^{\text {High }}$ SHG MK (purity shown in Online Supplementary Figure S3). Transmission electron microscopy revealed that PS ${ }^{\text {Low }}$ LLG MK exhibited many typical features of BM $\mathrm{MK}$, including large cells with multi-lobed nuclei, mitochondria, granules and surface protrusions (Figure 2A). In contrast, most of the PS High SHG MK were apoptotic, displaying membrane blebbing, highly condensed or absent nuclei, and few or no granules (Figure 2B). CD41a LLG $\mathrm{MK}$ showed a substantial increase in mitochondrial membrane potential compared to $\mathrm{CD}_{41 \mathrm{a}^{+}} \mathrm{SHG} \mathrm{MK}$ (Figure 1D), further supporting viability of the former.

Several approaches to characterize the functionality of the LLG and SHG MK were undertaken. The signaling capacity of these different MK populations was assessed and LLG, but not SHG, were able to activate integrin $\alpha I I b \beta 3$ in response to agonist stimulation (Figure 3A). In addition, thrombin-induced $\mathrm{Ca}^{2+}$ mobilization was observed only in LLG cells but not in SHG cells (Figure 3B). Importantly, by day 13 we observed MK proplatelet formation (PPF). After separating LLG from SHG based on PS exposure, we observed more PPF in PS ${ }^{\text {Low }}$ LLG MK than in PS ${ }^{\text {High }}$ SHG MK (Figure 3C).

Next, we sought to address whether the LLG population was the origin of the SHG cells. Day 13 PS ${ }^{\text {Low }}$ LLG MK were isolated and labeled with lipophilic cell tracking dye $\mathrm{DiO}^{18,19}$ and cultured for an additional 48 hours (h) to follow the 'movement' of the DiO label to SHG MK (Figure 3D). We observed an increase in $\mathrm{DiO}^{+}$LLG MK at day 15 (Figure $3 \mathrm{E}$ ). More importantly for this experiment, significantly more SHG MK contained tracking dye after an additional two days in culture (Figure $3 \mathrm{E}$ ). These data indicate that $\mathrm{CB}$-derived $\mathrm{MK}$ cultures represent a continuum of cells transitioning from mature viable CD41 $\mathrm{a}^{\text {High }}$ CD42 $a^{\text {High }}$ PS $^{\text {Low }}$ functional LLG MKs into CD41a ${ }^{\text {Low }}$ CD42 ${ }^{\text {Low }}$ PS $^{\text {High }}$ apoptotic SHG MK.

BCL2L2 regulates megakaryocyte apoptosis and larger, lower granular megakaryocyte number

To begin to understand the molecular mechanisms regulating cultured $\mathrm{MK}$ apoptosis, we screened 24 genes with established roles in intrinsic and extrinsic apoptosis pathways whose expression changed as MK matured in culture. Because the percentages of SHG MK substantially increased between day 6 and day 13 (Online Supplementary Figure S4), we performed gene expression profiling on CD61-purified MK at these time points and observed a 3-fold and 12-fold increase, respectively, in BCL2L1 and $B C L 2 L 2$, two anti-apoptotic Bcl-2 family members (Figure 4A). BCL2L1 encodes Bcl-xL and has an established role
A

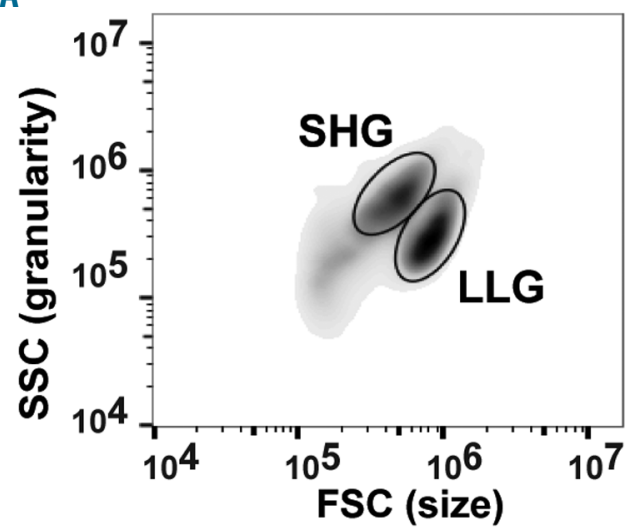

C

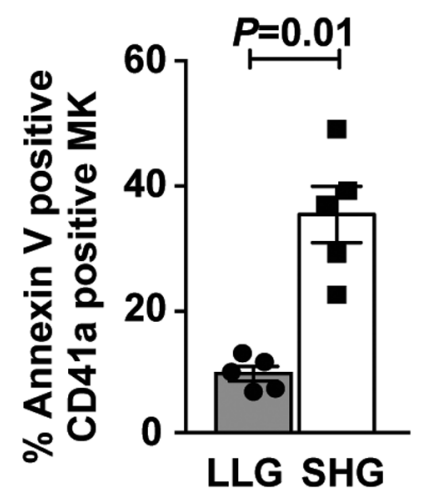

B

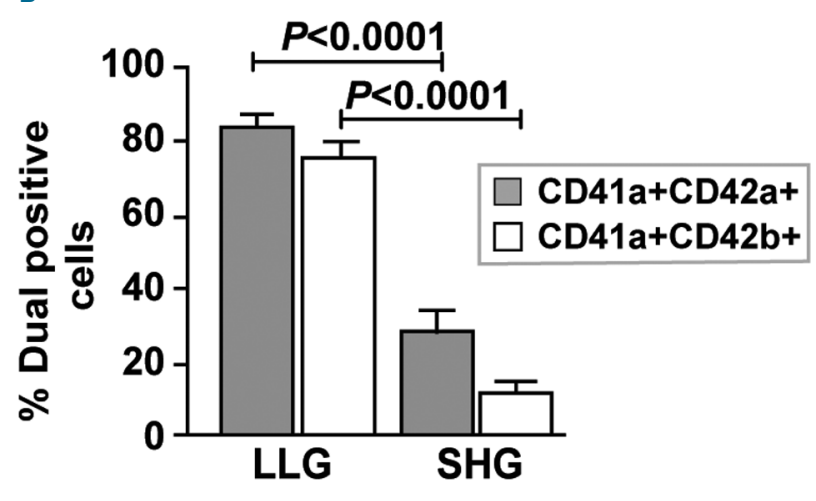

D

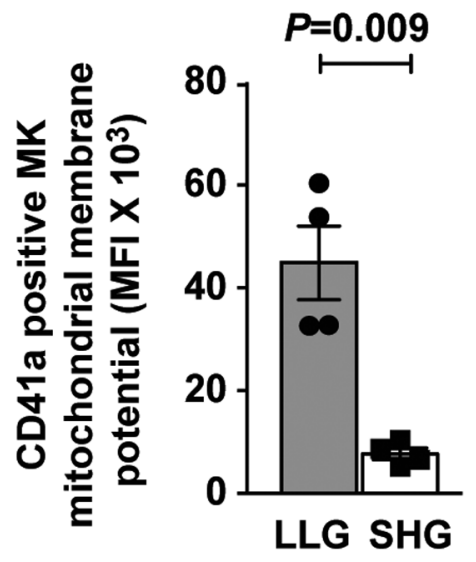

Figure 1. Two distinct populations emerge during cultured megakaryocytopoiesis. (A) Forward scatter (FSC) and side scatter (SSC) flow cytometric analysis of day 13 cultures showing the distinct larger size, lower granularity (LLG) and smaller size, higher granularity (SHG) populations of megakaryocytes (MK). (B) LLG and SHG cells were quantified for dual MK markers CD41aCD42a $(n=10)$ and CD41aCD42b $(n=10)$ by flow cytometry. (C) LLG and SHG MK were analyzed by flow cytometry using PE-labeled annexin V and APC-labeled anti-CD41a $(n=5)$. (D) LLG and SHG MK were stained with APC-labeled anti-CD41a and TMRM to detect mitochondrial membrane potential and analyzed by flow cytometry $(n=4)$. Error bars indicate mean \pm SEM. All assays performed on day 13 cultures. 
A
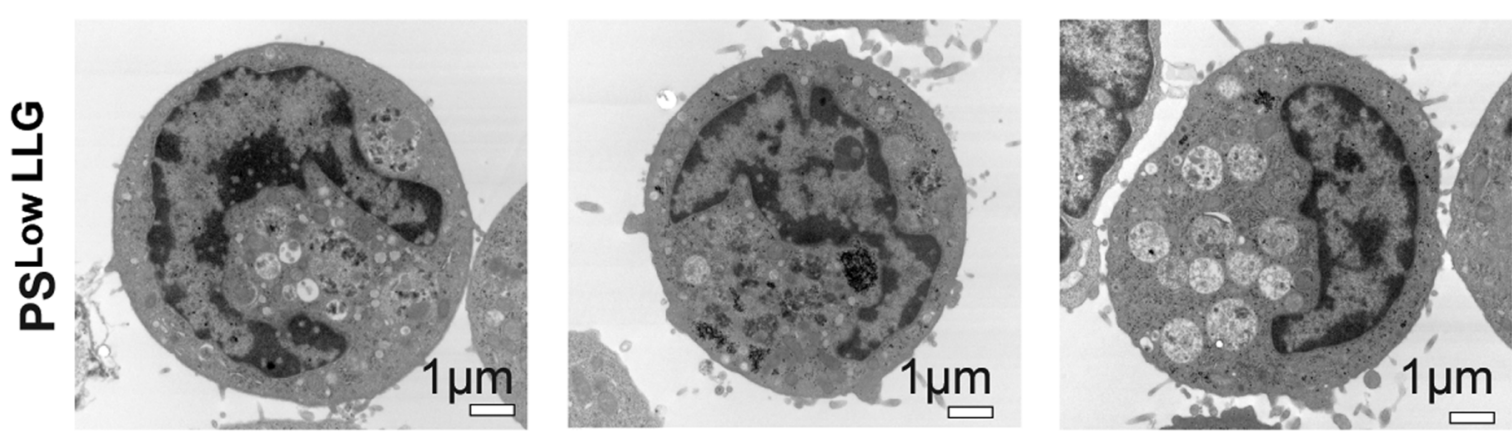

B
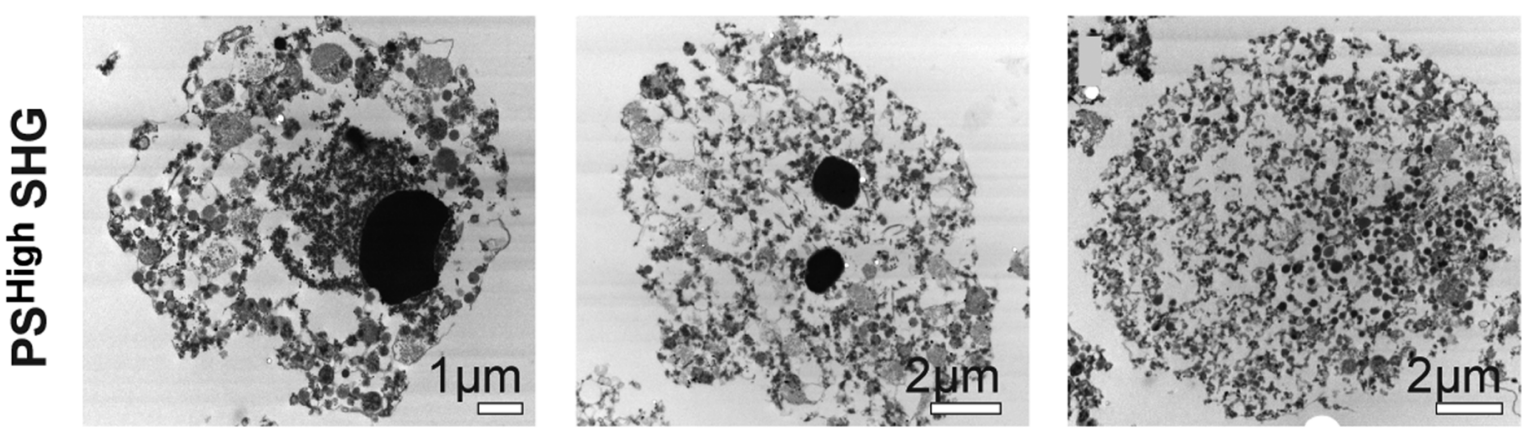

Figure 2. Transmission electron microscopy images of cultured megakaryocytes (MK). (A) Three representative annexin V microbead-isolated PS ${ }^{\text {Low }}$ larger size, lower granularity MK. (B) Three representative annexin V microbead-isolated PS ${ }^{\text {High }}$ smaller size, higher granularity MK.

A

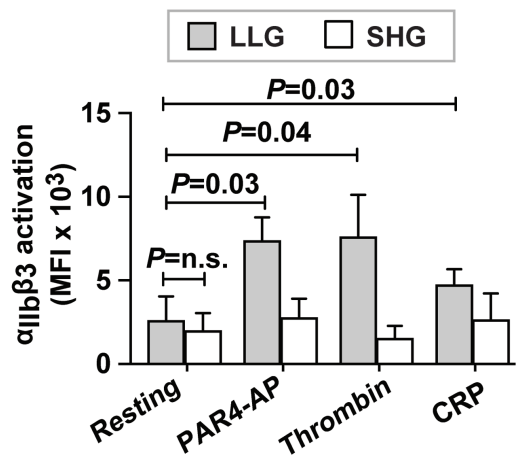

B

Thrombin-activated $=$ Resting
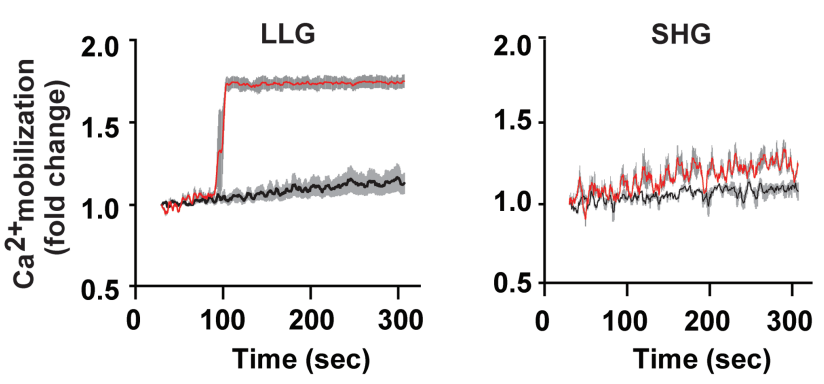

C

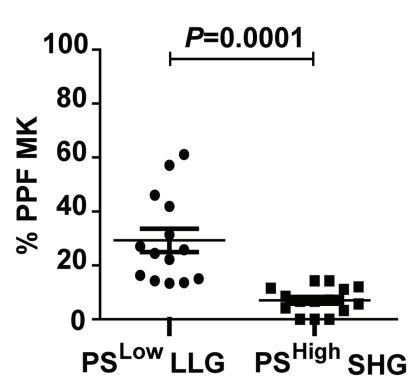

D

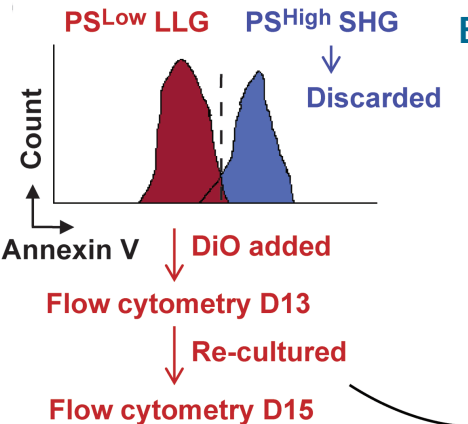

E $\square$ PS Low LLG $\square$ PS High SHG

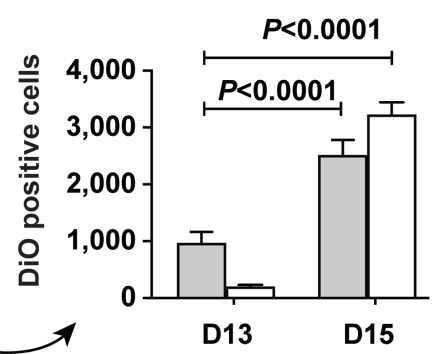

Figure 3. Larger size, lower granularity (LLG) and smaller size, higher granularity (SHG) megakaryocytes (MK) are viable and apoptotic, respectively. Assays performed on day 13 cultures for (A-C). (A) LLG and SHG MK were stimulated with PAR4-AP, thrombin, collagen related peptide (CRP) or no agonist (resting), and PAC1 binding (marker of integrin $\alpha$ llb $\beta 3$ activation) was quantified by flow cytometry $(n=4)$. n.s.: not significant. (B) LLG and SHG were stimulated with thrombin (red line) or no agonist (resting; black line) and calcium mobilization was measured (gray lines represent Standard Error of Mean for 3 separate experiments). (C) Quantification of proplatelet forming (PPF) MK blinded as to whether samples were PS ${ }^{\text {Low }}$ LLG or PS High SHG cells. Data were collected from five independent cords and each data point represents the percentage of PPF MK counted from randomly selected objective field image. An average 200 cells were counted per cord sample. (D) Schematic outlining for DiO labeling experiment. Day 13 PS ${ }^{\text {Low }}$ LLG MK were separated from PS ${ }^{\text {High }}$ SHG MKs using annexin V microbeads. PS Low LLG MK were labeled with Vybrant DiO cell tracking dye and re-cultured for two days. (E) Bar graph showing numbers of DiO-positive PS ${ }^{\text {Low }}$ LLG MKs and PS High SHG MK at day 13 and day 15 (48 hours post $\mathrm{DiO}$ addition) $(n=6)$. 
in MKpoiesis. ${ }^{12,13,20}$ However, BCL2L2 (encoding Bcl-w) has not been studied in Mkpoiesis and became the focus of further investigation. The increase in expression of BCL2L2 observed by RNA sequencing was verified by qPCR on RNA isolated from CD61-purified MK (Figure $4 \mathrm{~B})$. This led to the hypothesis that those MK with increasing levels of anti-apoptotic BCL2L2 would be the LLG, whereas those MK with stable or decreasing levels of anti-apoptotic BCL2L2 would be the SHG. LLG MK and SHG MK were purified by cell sorting and analyzed for changes in BCL2L2 expression over time in culture. There was no difference between LLG and SHG cells for BCL2L2 expression at days 6 and 9, but a significant difference was observed by day 13 due to a fall in BCL2L2 in SHG cells and concomitant rise in LLG cells (Figure 4C).

We next considered the effects of BCL2L2 on cultured $\mathrm{MK}$ apoptosis and number. Initially, we used the general Bcl-2 family inhibitor, ABT-263, to screen for an effect on CD41a ${ }^{+}$LLG MK numbers, and observed a significant reduction (Online Supplementary Figure S5). Since ABT-263 inhibits all Bcl-2 family members, we specifically tested the effects of BCL2L2 on the cultured MK. CB-derived $\mathrm{MK}$ cultures were transduced with lentiviral vectors containing BCL2L2, and both mRNA and protein increased by day 13 (Figure 5A-C). BCL2L2 overexpression significantly reduced the percentage of annexin $\mathrm{V}^{+} \mathrm{CD} 41 \mathrm{a}^{+} \mathrm{MK}$ (Figure 5D) and increased the number of CD41a LLG MK by $19 \%\left(1.36 \times 10^{5}\right.$ to $\left.1.61 \times 10^{5} ; P=0.049\right)$.
$B C L 2 L 2$ regulates megakaryocyte proplatelet formation

Megakaryocyte PPF is believed to be a critical process in thrombopoiesis. By day 13 we observed MK PPF primarily in LLG MK (Figure 3C), and asked whether Bcl-w might affect PPF. Importantly, when we scored MK PPF blinded to lentiviral transduction treatment group, we found that BCL2L2 overexpression induced a significant 58\% increase in PPF MK (Figures 5E and F).

\section{Relationship between BCL2L2 expression and platelet number}

Megakaryocyte PPF is tightly linked to $\mathrm{PB}$ platelet count, ${ }^{10,21,22}$ raising the possibility that BCL2L2 may regulate platelet number as well as MKpoiesis. Because we had previously performed genome-wide platelet gene expression profiling in 154 healthy individuals, ${ }^{14}$ we were able to query this dataset for such an association. Platelet BCL2L2 mRNA levels were positively correlated with platelet count in the PRAX1-1 study (Figure 6A).

As is typical of MK culture systems, the day 13 cultures also contained a population of small, hypogranular particles that overlapped the forward and side scatter properties of normal human platelets (Figure 6B). These particles are typically referred to as platelet-like particles (PLP), and we assessed the effect of MK BCL2L2 overexpression on PLP number and function. Compared to control lentiviral transduction, BCL2L2 overexpression induced a modest but significant increase in CD41a+ PLP from approximate-

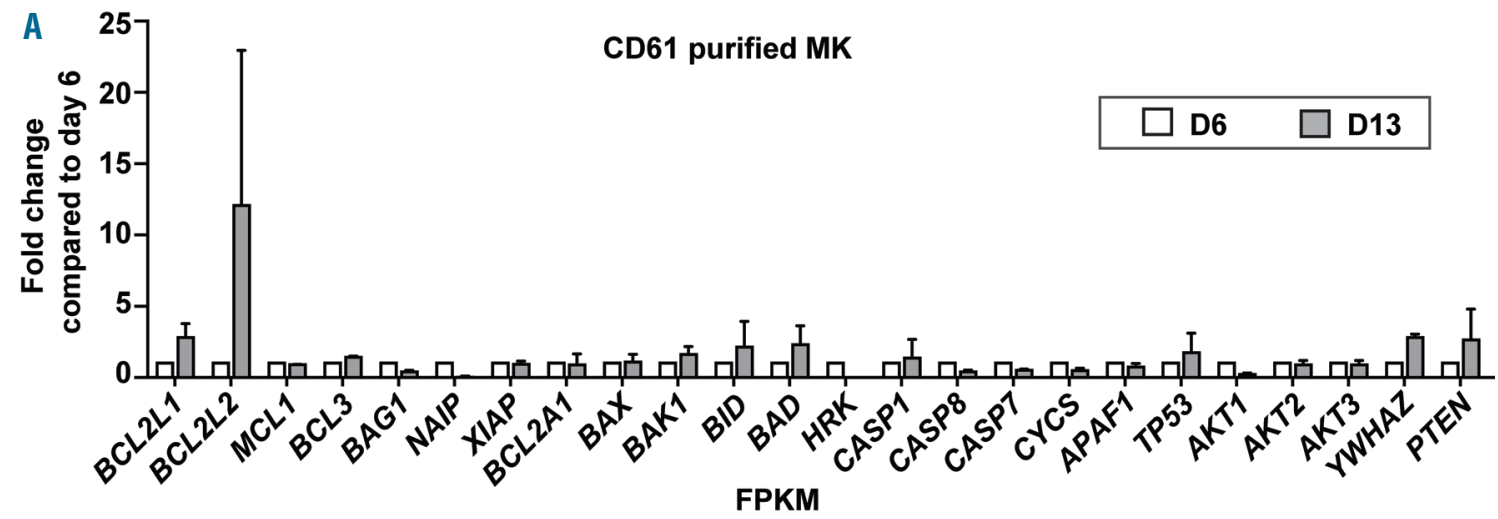

B

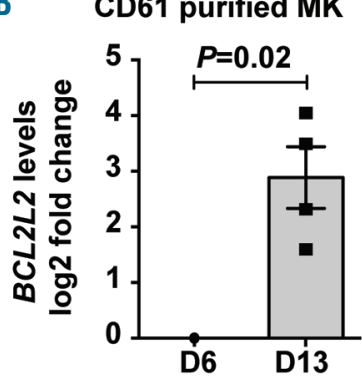

C

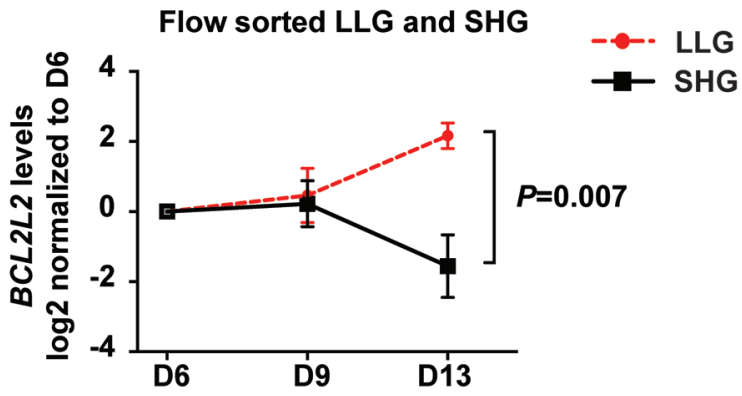

Figure 4. Expression of the anti-apoptotic gene BCL2L2 increases functional megakaryocytes (MK). CD61+ MK RNA was isolated from day 6 and day 13 cultures for analyses in (A) and (B). (A) CD61-positive $\left(^{+}\right)$MK expression of 24 apoptosis-related genes was assessed by RNA sequencing $(n=3)$. Average fold changes in expression for day 13 versus day 6 are shown. (B) CD61+ MK BCL2L2 quantification by quantitative polymerase chain reaction (qPCR) ( $=4)$. (C) Larger size, lower granularity (LLG) and smaller size, higher granularity (SHG) MK were flow cytometrically sorted and BCL2L2 expression was quantified by qPCR. Fold changes were determined for LLG and SHG MK compared to their respective day 6 expression ( $n=4$ for day 6 and day $13 ; n=6$ for day 9 ). 
ly $10 \%$ to approximately $19 \%$ (Figure 6C), suggesting BCL2L2 enables mature platelet production.

Platelet-like particles were stimulated with thrombin to assess their ability to activate $\alpha \operatorname{Ilb} \beta 3$ and release $\alpha$-granules. PLP from both control and BCL2L2 lentiviral constructs demonstrated cell activation above background (Figure 5D and E, and flow cytometry plots in Online Supplementary Figure S6); the signal was small but consistent in PLP with control lentivirus. Importantly, among these CD41a $\mathrm{a}^{+}$and CD42a $\mathrm{a}^{+}$PLP, MK BCL2L2 overexpression enhanced thrombin-induced activation compared to control lentivirus (Figure 6D and E, and flow cytometry plots in Online Supplementary Figure S6). PAC1 binding and P-selectin expression in PLP treated with buffer instead of thrombin were not altered by BCL2L2 overexpression (data not shown).

\section{Discussion}

There has been exciting progress in the generation of $\mathrm{MK}$ and platelets via the use of induced pluripotent stem cells, immortalized MK cell lines, and bioreactors, ${ }^{1,23,24}$ but cultured human CB MK remain an important tool for gaining a deeper understanding of MKpoiesis and platelet production. Apoptosis of $M K$ in a culture system remains a major obstacle to progress in the in vitro generation of $\mathrm{MK}$ and platelets. ${ }^{6}$ A great deal of our understanding about the apoptosis genes regulating MKpoiesis and $\mathrm{PB}$ platelet number is derived from work in murine systems, ${ }^{12,20,25-27}$ while relatively less is known about in vitro cultured $\mathrm{MK}$ apoptosis. The major finding in the current study is that the pro-survival gene $B C L 2 L 2$ restrains apoptosis in cultured human MK, regulates PPF, and is associated with platelet number in healthy humans. We also found that suspensions of $\mathrm{CB}$-derived $\mathrm{MK}$ are a useful system for assessing candidate gene function by flow cytometric analysis, and that BCL2L2 overexpression induced increases in agonist-induced signaling responses in PLP. BCL2L2 thus becomes another potential target for enhancing $\mathrm{MK}$ yields in vitro, which may benefit both basic research on MKpoiesis and the long-term goal of producing platelets for transfusion into patients.

Two distinct populations of cells invariably emerged during our $\mathrm{CB}$ MK cultures that were easily distinguished by flow cytometry logarithmic scale forward and side scatter measures. Electron microscopic analysis of the LLG $\mathrm{MK}$ (Figure 2) demonstrated they were most similar to primary BM MK, and resembled those observed by Cramer et al..$^{28}$ and Chantelot-Bellanne et al. ${ }^{29}$ In contrast, the SHG population represented primarily apoptotic cells resembling the senescent MK observed by Radley et al., where the nucleus fragmented but the cells appeared to change shape and 'round up' rather than fragment. ${ }^{30}$ Membrane degradation during apoptosis likely contributed to the loss of MK markers (Figure 1B). The most important functional differences between LLG and SHG were the ability to form proplatelets and 'signal' in response to platelet ago-
A

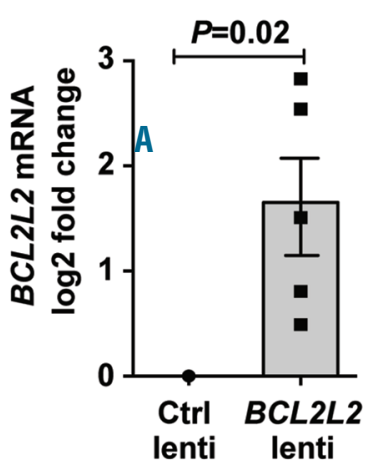

D

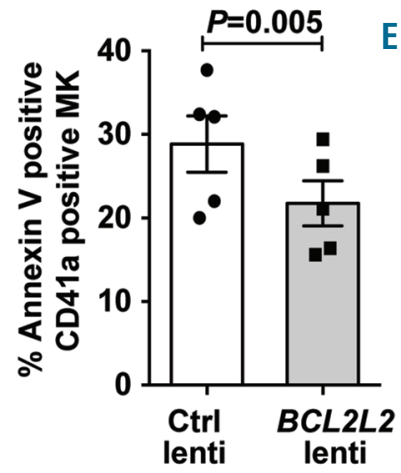

B
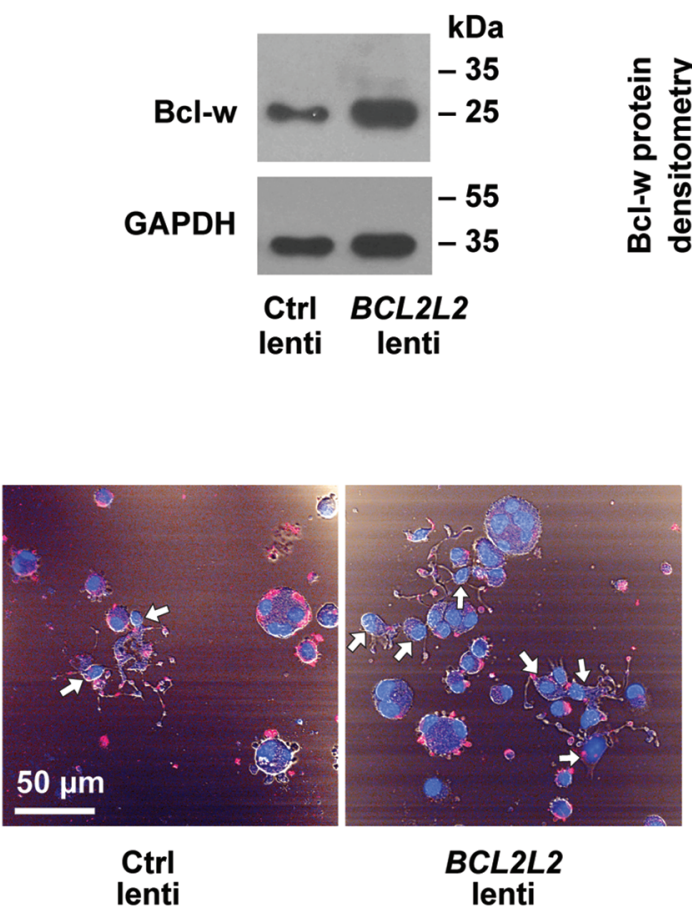
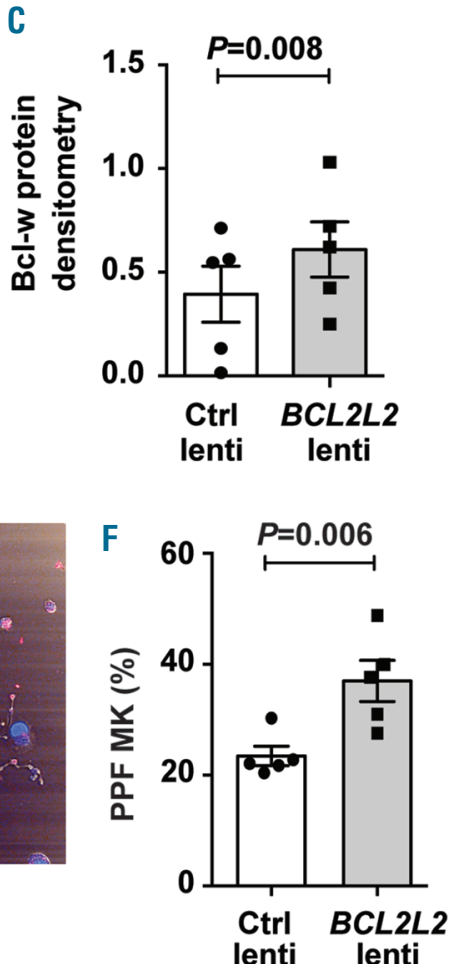

Figure 5. BCL2L2 overexpression reduces megakaryocyte (MK) apoptosis. (A-F) Transduction of MK cultures with empty lentiviral vector (Ctrl lenti) or lentiviral vector containing BCL2L2. (A) Overexpression of BCL2L2 mRNA was confirmed by quantitative polymerase chain reaction (qPCR). Fold change of $B C L 2 L 2$ mRNA levels was determined compared to Ctrl lenti $(n=5)$. (B) Representative western blot image for Bcl-w protein (encoded by BCL2L2 gene) for BCL2L2 overexpression compared to Ctrl. (C) Densitometric analysis of western blots for BCL2L2 overexpression compared to Ctrl ( $n=5)$. Glyceraldehyde-3-phosphate dehydrogenase (GAPDH) was used as a normalizer for densitometric analysis. (D) The percentage of annexin V-positive $\left(^{+}\right)$CD41a ${ }^{+}$MK was determined for BCL2L2 over-expressed and Ctrl cells by flow cytometry $(n=5)$. (E) Representative image of proplatelet formation in Ctrl and BCL2L2 over-expressed cells. Image shows $\alpha$-tubulin staining (red) and nuclear stain, DAPI (blue). White arrows show proplatelet-forming MK. (F) Quantification of proplatelet forming (PPF) MK amongst total cells blinded to treatment group ( $n=5$ ). 
nists (Figure 2A-C). It is worth noting that although PSpositivity marked apoptotic SHG MK, some fraction of LLG MK were also capable of PS exposure in response to thrombin stimulation (Online Supplementary Figure S7), perhaps akin to collagen and thrombin activated (COAT) platelets. ${ }^{31-33}$ Thus, simple gating on flow cytometric forward and side scatter measurements, without the need for fluorescently-labeled antibodies, identified functional MK and can improve signal-to-noise read-outs that test the functionality of candidate $\mathrm{MK} /$ platelet genes.

Apoptosis was established as a mechanism for platelet lifespan by the Kile laboratory when they showed that the balance between Bcl-xL and Bak regulate murine lifespan in vivo. ${ }^{20}$ The pro-survival Bcl-2 family member Bcl-xL degrades in aging platelets, thus allowing apoptosis to proceed. In addition to Bcl-xL, the Bcl-2 family includes Bcl2, Mcl-1 and Bcl-w, which are encoded by BCL2, MCL1 and BCL2L2, respectively. Altered murine expression of pro-survival Bcl-2 family members has shown variable effects on platelet number. Global chimeric vav-Bcl2 overexpression resulted in normal MK numbers but an approximately $50 \%$ reduction in platelet counts, ${ }^{34}$ whereas MKspecific deletion of $B c l 2$ had no effect on platelet number. ${ }^{35}$ MK-specific deletion of Bcl2l1 deletion caused MK apoptosis, loss of platelet shedding, and a macrothrombocytopenia. ${ }^{12}$ MK-specific deletion of Mcll did not affect MK number or morphology, or platelet count or volume. ${ }^{13}$ Print et al. globally inactivated $B c l 2 l 2$ to study its importance in murine physiology. ${ }^{36}$ Although Mkpoiesis was not a major focus of their study, they reported that three
$\mathrm{BCl}_{2} 12^{-}$mice displayed numbers of $\mathrm{MK}$ colony-forming cells and platelets comparable to three wild-type littermates. This latter report appears to differ from our finding in primary human $\mathrm{MK}$ and platelets, where BCL2L2 increased during $M K$ differentiation, overexpression of BCL2L2 increased the numbers of both the viable CD41a LLG MK and MK PPF (Figure 4E and F), and BCL2L2 levels correlated with platelet number (Figure 5A). This apparent discrepancy between human and mouse regarding the effect of BCL2L2 on MKpoiesis and platelet production could be due to a compensatory upregulation of other Bcl2 family members in the Bcl2l2 null mice. Alternatively, small numbers of mice and different conditions may have led to a chance finding in the Print et al. study, ${ }^{36}$ but there also may be species differences in the relative importance of BCL2L2 because human platelets contain 3.6-fold higher levels of BCL2L2 transcripts than mouse platelets. ${ }^{37}$

We showed that the SHG MK were derived from the LLG MK (Figure 2D and E), supporting a process by which viable LLG avoid apoptosis long enough to mature and acquire mature $\mathrm{MK}$ markers, but an unknown trigger induces some cells to become dying SHG. The day 9 to day 13 increase in BCL2L2 is expected to restrain apoptosis in LLG cells, whereas BCL2L2 reduction should enable apoptosis to proceed in SHG cells (Figure 3C). Perhaps a yet-to-be-defined switch regulates BCL2L2 levels after day 9 in cultured cells. CB-derived LLG MK began to develop proplatelet extension around day 12 and peaked at days 13-15, similar to what had been reported by Balduini et al. ${ }^{38}$ Because SHG appear days before PPF MK (Online
A

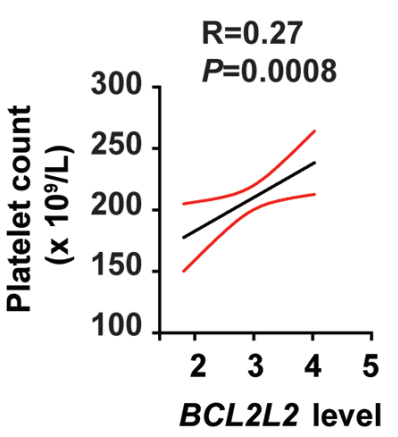

C

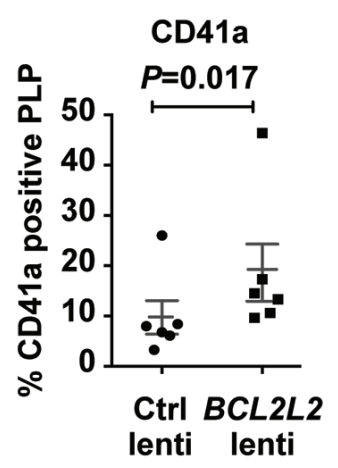

B

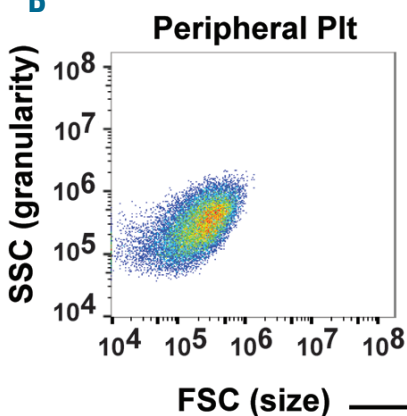

D

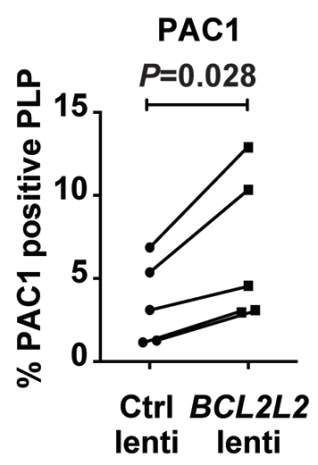

d13 PLP

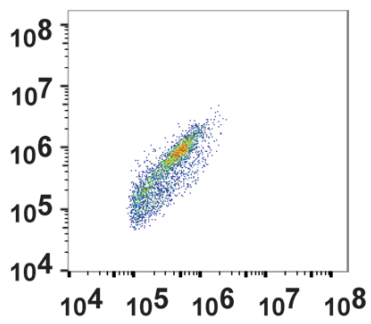

Merged

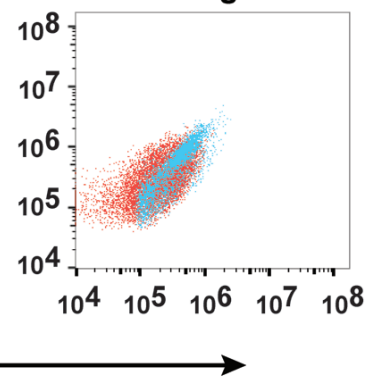

E

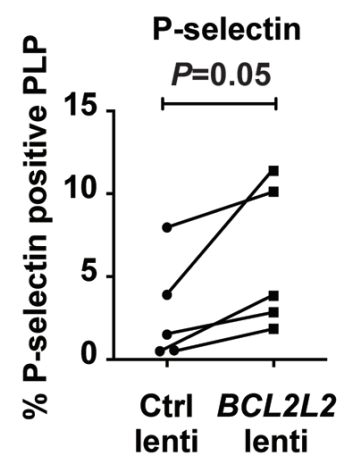

Figure 6. Relationship of BCL2L2 to blood platelet number and cultured platelet-like particles (PLP). (A) BCL2L2 is positively associated with peripheral blood platelet counts in 154 healthy individuals in the PRAX1 study. (B) Forward scatter and side scatter flow cytometric analysis of human peripheral blood platelets (Peripheral PIts), day 13 PLP and the merged image. (C-E) Flow cytometric assessment of cultured PLP after MK transduction with empty lentiviral vector (Ctrl lenti) or lentiviral vector containing BCL2L2. PLP were defined by forward scatter, side scatter and presence of platelet-specific surface marker (see Methods). (C) The percentages of day 13 PLP that were CD41a-positive $\left(^{+}\right)(n=6)$. (D) The percentages of day 13 PLP that bound PAC1 after stimulation with $250 \mathrm{nM}$ thrombin ( $\left.n=5\right)$. (E) The percentages of day 13 PLP that expressed P-selectin after stimulation with $250 \mathrm{nM}$ thrombin $(n=5)$. 
Supplementary Figure S4), they do not seem to be 'exhausted' MK post release of proplatelets.

Sim et al. used adult mobilized HSPC to culture MK that also gave rise to populations of functional and apoptotic MK. ${ }^{17}$ Our work differs from this primarily by studying the molecular mechanism of apoptosis. We are unaware of direct comparisons among any source of MK (neonatal, adult, murine, embryonic stem cells, etc.) in terms of the degree of apoptosis in culture, but it is reasonable to consider that some molecular mechanisms will be shared. In this regard, our data show no statistically significant difference between BCL2L2 levels in neonatal and adult MK $(P>0.47)$, and adult MK also display more PPF with BCL2L2 overexpression (Online Supplementary Figure S8). As with $\mathrm{MK}$, there is no statistically significant difference between BCL2L2 levels in neonatal and adult platelets. ${ }^{39}$

The finding of a significant positive correlation between human platelet BCL2L2 levels and PB platelet counts (Figure 5A) supports a mechanism whereby Bcl-w increases MK PPF which causes increased blood platelets. Numerous apoptosis genes have been studied for effects on Mkpoiesis. ${ }^{8-13,19,40,41}$ We observed that pro-apoptotic genes $B A X$ and $B A K 1$ showed little to no change in expression from day 6 to day 13 in MK cultures (Online Supplementary Figure $S 9 A$ and B). However, similar to $B C L 2 L 2$, anti-apoptotic gene BCL2L1 showed increased expression in LLG over time, and was higher at day 13 in LLG compared to SHG (Online Supplementary Figure S9C). Taken together, these studies suggest BCL2L1 and BCL2L2 restrain apoptosis and that this may be the key mechanism for prolonging $\mathrm{MK}$ survival in the late stages of differentiation in culture. We cannot exclude a role for other apoptosis genes in Mkpoiesis and platelet production. It is also reasonable to consider that, like $\mathrm{Bcl}-\mathrm{xL}$ and Bak, Bcl$\mathrm{W}$ may also impact the life span of the human PB platelet. ${ }^{20}$ However, additional studies are needed to address this possibility.

The BCL2L2-induced increase in MK PPF led to experiments assessing production of PLP. BCL2L2 overexpression roughly doubled the number of $\mathrm{CD}_{4} 1 \mathrm{a}^{+}$PLP and increased the ability of these PLP to show a functional response when activated with thrombin (Figure 5C-E). However, we did not observe any significant correlation between in vivo BCL2L2 expression with other platelet agonists such as ADP, PAR1-AP and PAR4-AP ( $w$ ww . Plateletomics.com, as reported by Simon et $a{ }^{40}{ }^{40}$ ). Nevertheless, we would emphasize that the percentages of functional PLP were small, although consistent and statistically significant.

Our findings have several research and clinical implications. Firstly, we have shown that simple gating on flow cytometric forward and side scatter measurements allows the identification of the subset of viable and functional MK. This approach represents a substantial improvement in the signal-to-noise read-outs to test the functionality of candidate $\mathrm{MK} /$ platelet genes identified in GWAS. Secondly, promotion of apoptosis by inhibiting of Bcl-2 family members is under investigation for the treatment of a variety of malignancies; ${ }^{41-43}$ a limiting toxicity has been thrombocytopenia. ${ }^{42,43}$ Bcl-2 family members, MCL1 and BCL2L1 are the most frequently amplified in 26 tumor types, ${ }^{44,45}$ whereas BCL2L2 amplification is rare. ${ }^{46}$ Perhaps selective targeting of $\mathrm{Bcl}-2$ family members that spares $\mathrm{Bcl}-\mathrm{w}$ could minimize thrombocytopenia. Lastly, progress toward the manufacture of in vitro platelets may benefit from the pro-survival benefits on MK generation and PPF of Bcl-w. BCL2L2 overexpression may have synergistic or additive effects when used in combination with an antagonist of the aryl hydrocarbon receptor (StemRegenin 1), BCL2L1 overexpression, abscisic acid, and other factors that have been shown to increase numbers of proplateletforming $\mathrm{MK}$ in culture. ${ }^{1,47,48}$

\section{Funding}

This study was supported by a grant from the National Institutes of Health National Heart, Lung and Blood Institute (HL116713) and funding from the Cardeza Foundation of Hematologic Research. The authors thank Ms. Lin Ma, Thomas Jefferson University, and Ms. Sarah Hugo, University of Utah for their help isolating cord blood stem cells; Dr. Xianguo Kong, Thomas Jefferson University for designing lentiviral vectors; Ms. Diana Lim, Molecular Medicine Program from University of Utah, for drafting the figures and consultation on data display.

\section{Acknowledgments}

The authors also thank the University of Utah Flow Cytometry Facility in addition to the National Cancer Institute through Award Number 5P30CA042014-24.

\section{References}

1. Ito $\mathrm{Y}$, Nakamura $\mathrm{S}$, Sugimoto $\mathrm{N}$, et al. Turbulence Activates Platelet Biogenesis to Enable Clinical Scale Ex Vivo Production. Cell. 2018;174(3):636-648.e18.

2. Moreau T, Evans AL, Vasquez L, et al. Largescale production of megakaryocytes from human pluripotent stem cells by chemically defined forward programming. Nat Commun. 2016;7:11208.

3. Nakamura S, Takayama N, Hirata S, et al. Expandable megakaryocyte cell lines enable clinically applicable generation of platelets from human induced pluripotent stem cells. Cell Stem Cell. 2014;14(4):535-548.

4. Thon JN, Dykstra BJ, Beaulieu LM. Platelet bioreactor: accelerated evolution of design and manufacture. Platelets. 2017;28(5):472477.
5. Zhang $\mathrm{N}$, Zhi $\mathrm{H}$, Curtis $\mathrm{BR}$, et al. CRISPR/Cas9-mediated conversion of human platelet alloantigen allotypes. Blood. 2016;127(6):675-680.

6. Zauli G, Vitale M, Falcieri E, et al. In vitro senescence and apoptotic cell death of human megakaryocytes. Blood. 1997;90(6): 2234-2243.

7. Kile BT. The role of apoptosis in megakaryocytes and platelets. Br J Haematol. 2014; 165(2):217-226

8. Morison IM, Cramer Borde EM, Cheesman EJ, et al. A mutation of human cytochrome c enhances the intrinsic apoptotic pathway but causes only thrombocytopenia. Nat Genet. 2008;40(4):387-389.

9. Kaluzhny Y, Yu G, Sun S, et al. BclxL overexpression in megakaryocytes leads to impaired platelet fragmentation. Blood. 2002;100(5):1670-1678.
10. De Botton S, Sabri S, Daugas E, et al. Platelet formation is the consequence of caspase activation within megakaryocytes. Blood. 2002;100(4):1310-1317.

11. Kodama T, Takehara T, Hikita H, et al. BH3only activator proteins Bid and Bim are dispensable for Bak/Bax-dependent thrombocyte apoptosis induced by Bcl-xL deficiency: molecular requisites for the mitochondrial pathway to apoptosis in platelets. J Biol Chem. 2011;286(16):13905-13913.

12. Josefsson EC, James C, Henley KJ, et al. Megakaryocytes possess a functional intrinsic apoptosis pathway that must be restrained to survive and produce platelets. J Exp Med. 2011;208(10):2017-2031.

13. Debrincat MA, Josefsson EC, James C, et al. Mcl-1 and Bcl-x(L) coordinately regulate megakaryocyte survival. Blood. 2012; 119(24):5850-5858. 
14. Edelstein LC, Simon LM, Montoya RT, et al Racial differences in human platelet PAR4 reactivity reflect expression of PCTP and miR-376c. Nat Med. 2013;19(12):1609-1616.

15. Faraday N, Rade JJ, Johns DC, et al. Ex vivo cultured megakaryocytes express functional glycoprotein IIb-IIIa receptors and are capable of adenovirus-mediated transgene expression. Blood. 1999;94(12):4084-4092.

16. Bergmeier W, Burger PC, Piffath CL, et al. Metalloproteinase inhibitors improve the recovery and hemostatic function of in vitro-aged or -injured mouse platelets. Blood. 2003;102(12):4229-4235.

17. Sim X, Jarocha D, Hayes V, et al. Identifying and enriching the platelet-producing human stem cell-derived megakaryocytes using factor V uptake. Blood. 2017;130(2):192204.

18. Parish CR. Fluorescent dyes for lymphocyte migration and proliferation studies. Immunol Cell Biol. 1999;77(6):499-508.

19. Ragnarson B, Bengtsson L, Haegerstrand A. Labeling with fluorescent carbocyanine dyes of cultured endothelial and smooth muscle cells by growth in dye-containing medium. Histochemistry. 1992;97(4):329-333.

20. Mason KD, Carpinelli MR, Fletcher JI, et al. Programmed anuclear cell death delimits platelet life span. Cell. 2007;128(6):11731186.

21. Beauchemin H, Shooshtarizadeh P, Vadnais C, Vassen L, Pastore YD, Moroy T. Gfilb controls integrin signaling-dependent cytoskeleton dynamics and organization in megakaryocytes. Haematologica. 2017;102 (3):484-497.

22. Machlus KR, Johnson KE, Kulenthirarajan R, et al. CCL5 derived from platelets increases megakaryocyte proplatelet formation. Blood. 2016;127(7):921-926.

23. Thon JN, Mazutis L, Wu S, et al. Platelet bioreactor-on-a-chip. Blood. 2014; 124(12): 1857-1867.

24. Sugimoto N, Eto K. Platelet production from induced pluripotent stem cells. J Thromb Haemost. 2017;15(9):1717-1727.

25. McArthur K, Chappaz S, Kile BT. Apoptosis in megakaryocytes and platelets: the life and death of a lineage. Blood. 2018;131(6):605610.

26. Schoenwaelder SM, Jarman KE, Gardiner $\mathrm{EE}$, et al. Bcl-xL-inhibitory $\mathrm{BH} 3$ mimetics can induce a transient thrombocytopathy that undermines the hemostatic function of platelets. Blood. 2011;118(6):1663-1674

27. White MJ, Schoenwaelder SM, Josefsson EC, et al. Caspase- 9 mediates the apoptotic death of megakaryocytes and platelets, but is dispensable for their generation and function. Blood. 2012;119(18):4283-4290.

28. Cramer EM, Norol F, Guichard J, et al. Ultrastructure of platelet formation by human megakaryocytes cultured with the Mpl ligand. Blood. 1997;89(7):2336-2346.

29. Bellanne-Chantelot C, Schmaltz-Panneau B, Marty C, et al. Mutations in the SRP54 gene cause severe congenital neutropenia as well as Shwachman-Diamond-like syndrome. Blood. 2018;132(12):1318-1331.

30. Radley JM, Haller CJ. Fate of senescent megakaryocytes in the bone marrow. Br J Haematol. 1983;53(2):277-287.

31. Alberio L, Safa O, Clemetson KJ, Esmon CT, Dale GL. Surface expression and functional characterization of alpha-granule factor $\mathrm{V}$ in human platelets: effects of ionophore A23187, thrombin, collagen, and convulxin. Blood. 2000;95(5):1694-1702.

32. Dale GL, Friese P, Batar P, et al. Stimulated platelets use serotonin to enhance their retention of procoagulant proteins on the cell surface. Nature. 2002;415(6868):175179.

33. Jobe SM, Leo L, Eastvold IS, et al. Role of FcRgamma and factor XIIIA in coated platelet formation. Blood. 2005; 106(13): 4146-4151.

34. Ogilvy S, Metcalf D, Print CG, Bath ML, Harris AW, Adams JM. Constitutive Bcl-2 expression throughout the hematopoietic compartment affects multiple lineages and enhances progenitor cell survival. Proc Natl Acad Sci U S A. 1999;96(26):14943-14948.

35. Debrincat MA, Pleines I, Lebois $M$, et al. BCL-2 is dispensable for thrombopoiesis and platelet survival. Cell Death Dis. 2015;6:e1721.

36. Print CG, Loveland KL, Gibson L, et al Apoptosis regulator bcl-w is essential for spermatogenesis but appears otherwise redundant. Proc Natl Acad Sci U S A. 1998; 95(21):12424-12431.

37. Rowley JW, Oler AJ, Tolley ND, et al Genome-wide RNA-seq analysis of human and mouse platelet transcriptomes. Blood. 2011;118(14):e101-111.

38. Balduini A, Pallotta I, Malara A, et al
Adhesive receptors, extracellular proteins and myosin IIA orchestrate proplatelet formation by human megakaryocytes. Thromb Haemost. 2008;6(11):1900-1907.

39. Caparros-Perez E, Teruel-Montoya R, Lopez-Andreo MJ, et al. Comprehensive comparison of neonate and adult human platelet transcriptomes. PLoS One. 2017; 12(8):e0183042.

40. Simon LM, Edelstein LC, Nagalla $S$, et al Human platelet microRNA-mRNA networks associated with age and gender revealed by integrated plateletomics. Blood. 2014;123(16):e37-45.

41. Oltersdorf T, Elmore SW, Shoemaker AR, et al. An inhibitor of Bcl-2 family proteins induces regression of solid tumours. Nature. 2005;435(7042):677-681.

42. Roberts AW, Seymour JF, Brown JR, et al Substantial susceptibility of chronic lymphocytic leukemia to BCL2 inhibition: results of a phase I study of navitoclax in patients with relapsed or refractory disease. J Clin Oncol. 2012;30(5):488-496.

43. Wilson WH, O'Connor OA, Czuczman MS et al. Navitoclax, a targeted high-affinity inhibitor of BCL-2, in lymphoid malignancies: a phase 1 dose-escalation study of safety, pharmacokinetics, pharmacodynamics, and antitumour activity. Lancet Oncol. 2010 11(12):1149-1159.

44. De Blasio A, Vento R, Di Fiore R. Mcl-1 targeting could be an intriguing perspective to cure cancer. J Cell Physiol. 2018; 233(11):8482-8498.

45. Beroukhim R, Mermel CH, Porter D, et al The landscape of somatic copy-number alteration across human cancers. Nature. 2010;463(7283):899-905.

46. Cerami E, Gao J, Dogrusoz U, et al. The cBio cancer genomics portal: an open platform for exploring multidimensional cancer genomics data. Cancer Discov. 2012;2(5): 401-404.

47. Malara A, Fresia C, Di Buduo CA, et al. The Plant Hormone Abscisic Acid Is a Prosurvival Factor in Human and Murine Megakaryocytes. J Biol Chem. 2017;292 (8):3239-3251.

48. Strassel C, Brouard N, Mallo L, et al. Aryl hydrocarbon receptor-dependent enrichment of a megakaryocytic precursor with a high potential to produce proplatelets. Blood. 2016;127(18):2231-2240. 